\title{
EMBEDDINGS IN THE TRIVIAL RANGE ${ }^{1}$
}

\author{
BY HERMAN GLUCK ${ }^{2}$
}

Communicated by Edwin Moise, May 23, 1963

1. Introduction. The fundamental problem of embedding theory is the

EMBEDDING PROBLEM. Given a polyhedron $P^{k}$ and an n-manifold $M^{n}$, classify the nice embeddings of $P^{k}$ into $M^{n}$ under equivalence by (ambient) isotopy.

This problem projects in a natural way into the

HoMOTOPY PROBLEM. Given a polyhedron $P^{k}$ and an n-manifold $M^{n}$, classify the continuous maps of $P^{k}$ into $M^{n}$ under equivalence by homotopy.

It is the object of this note to report ${ }^{4}$ that in the so-called "trivial" range of dimensions, $2 k+2 \leqq n$, there is a local, topological definition of "nice embedding" for which the above projection is bijective, and hence for which the two problems are equivalent.

Corresponding equivalences are well known in piecewise linear and differential topology $[1 ; 2]$, and are proved there by means of general position arguments. In the unrestricted topological case, where one considers all possible embeddings of $P^{k}$ into $M^{n}$, the desired results are hopelessly false. For example [3], there are many nonequivalent embeddings of the closed interval $[0,1]$ into Euclidean $n$-space $R^{n}$, $n \geqq 3$.

The main theorems of the present paper are

THEOREM 1.1. Let $f$ be a locally tame embedding of the polyhedron $P^{k}$ into the combinatorial manifold $M^{n}$. If $2 k+2 \leqq n$, then for each $\epsilon>0$ there is an $\epsilon$-push $h$ of $\left(M^{n}, f\left(P^{k}\right)\right)$ such that

$$
h f: P^{k} \rightarrow M^{n}
$$

is piecewise linear with respect to arbitrary preassigned triangulations of $P^{k}$ and $M^{n}$.

THEOREM 1.2. If $2 k+2 \leqq n$, then for each $\epsilon>0$ there is $a \delta>0$ such that if $f$ and $f^{\prime}$ are any two locally tame embeddings of the polyhedron $P^{k}$

${ }^{1} \mathrm{~A}$ result similar to Theorem 8.1 of this paper has been obtained independently by Charles Greathouse (this Bulletin, pp. 820-823).

2 Research supported in part by U. S. Army Research Office (Durham).

3 Definitions will be found in $\$ 2$.

${ }^{4}$ Missing details will appear elsewhere. 
into the combinatorial manifold $M^{n}$ with $d\left(f, f^{\prime}\right)<\delta$, then there exists an e-push $h$ of $\left(M^{n}, f\left(P^{k}\right)\right)$ satisfying $h f=f^{\prime}$.

THEOREM 1.3. Let $f$ and $f^{\prime}$ be locally tame embeddings of the polyhedron $P^{k}$ into the combinatorial manifold $M^{n}$. If $2 k+2 \leqq n$ and $f$ is homotopic to $f^{\prime}$, then there is a homeomorphism $h$ of $M^{n}$ onto itself which is isotopic to the identity, such that $h f=f^{\prime}$.

The first and crucial step towards the above theorems was taken by Homma [4]. His results are stated in $\$ 4$, and his techniques have been absorbed with only slight changes into the proof of Theorem 3.4.

2. Definitions. $P^{k}$ will denote a $k$-dimensional polyhedron, i.e., a space homeomorphic to the underlying space of some $k$-dimensional finite simplicial complex, and $M^{n}$ will denote a space homeomorphic to the underlying space of some combinatorial $n$-manifold without boundary. By a triangulation of $M^{n}$ we will always mean a triangulation as a combinatorial manifold. No particular triangulations, however, will be distinguished for either $P^{k}$ or $M^{n}$. Whenever there is occasion for a metric on $M^{n}$, we insist that $M^{n}$ be complete in the metric and that sets of sufficiently small diameter be enclosable in an $n$-cell lying in $M^{n}$. This can always be achieved by using the barycentric metric obtained from some triangulation of $M^{n}$.

Now let $f: P^{k} \rightarrow M^{n}$ be an embedding. If there is a triangulation of $P^{k}$ such that for each point $x \in P^{k}$ there is an open neighborhood $U$ of $f(x)$ in $M^{n}$ and a triangulation of $U$ as a combinatorial manifold in terms of which $f$ is piecewise linear on some neighborhood of $x$, then we will say that $f$ is a locally tame embedding. Note that the triangulation of $U$ need not be related to any triangulation of $M^{n}$. Note also that once a triangulation is chosen for $P^{k}$, the same triangulation must be used for deciding whether $f$ is locally tame at each point $x \in P^{k}$. However, if another embedding $f^{\prime}$ is given, an entirely different triangulation of $P^{k}$ may be used to decide whether $f^{\prime}$ is locally tame. This raises the question as to whether an embedding which is locally tame with respect to one triangulation of $P^{k}$ will be locally tame with respect to any triangulation of $P^{k}$. This question is answered affirmatively in the trivial range, $2 k+2 \leqq n$, by Theorem 6.1 , as a corollary to Theorem 1.1 .

Now let $M$ be a topological manifold with a metric $d$, and $A$ a subset of $M$. If $\epsilon \geqq 0$, an $\epsilon$-push of $(M, A)$ is a homeomorphism $h$ of $M$ onto itself such that

(1) $h$ is an $\epsilon$-homeomorphism, i.e., $d(x, h(x)) \leqq \epsilon$ for all $x \in M$,

(2) $h / M-U_{\epsilon}(A)=1$, i.e., if $d(x, A) \geqq \epsilon$, then $h(x)=x$, 
(3) $h$ is $\epsilon$-isotopic to the identity under an isotopy which restricts to the identity on $M-U_{\epsilon}(A)$, i.e., each $h_{t}$ of the isotopy satisfies conditions (1) and (2) above.

An $\epsilon$-push is usually just what a topologist means when he says "give a little push."

3. Formal properties of embeddings. In this section, $X$ will denote a metric space and $A \subset X$ a subset whose closure $\bar{A}$ is compact. $M$ will denote a topological manifold with a complete metric $d$.

$\operatorname{Hom}(X, A ; M)$ will denote an arbitrary set of embeddings of $X$ into $M$, all of which agree on $X-A$. That is, if $f, g \in \operatorname{Hom}(X, A ; M)$, then $f / X-A=g / X-A$. If $f, g \in \operatorname{Hom}(X, A ; M)$, we define a distance function

$$
d(f, g)=\mathrm{LUB}_{x \in \bar{X}} d(f(x), g(x))=\mathrm{LUB}_{x \in \bar{A}} d(f(x), g(x)),
$$

which exists because $\bar{A}$ is compact. This distance function makes $\operatorname{Hom}(X, A ; M)$ into a metric space.

Definition 3.1. Let $F$ be a subset of $\operatorname{Hom}(X, A ; M)$ with the property that for each $g \in \operatorname{Hom}(X, A ; M)$ and each real number $\epsilon>0$, there is an $f \in F$ with $d(f, g)<\epsilon$. In accordance with standard terminology, we say that $F$ is dense in $\operatorname{Hom}(X, A ; M)$.

Definition 3.2. Let $F$ be a subset of $\operatorname{Hom}(X, A ; M)$ with the following property: for any $\epsilon>0$ there is a $\delta>0$ such that if $f, f^{\prime} \in F$ and $d\left(f, f^{\prime}\right)<\delta$, then there is an $\epsilon$-push $h$ of $(M, f(A))$ such that $h f=f^{\prime}$. Then we say that $F$ is solvable.

From this definition we easily obtain

Theorem 3.3. Let $F \subset F^{\prime} \subset \operatorname{Hom}(X, A ; M)$. Suppose that for each $f^{\prime} \in F^{\prime}$ and each $\epsilon>0$, there is an $\epsilon$-push $h$ of $\left(M, f^{\prime}(A)\right)$ such that $f=h f^{\prime}$ is an element of $F$. If $F$ is solvable, then so is $F^{\prime}$ solvable.

The main result is

THEOREM 3.4. The union of two dense, solvable subsets of $\operatorname{Hom}(X, A ; M)$ is dense and solvable.

The proof of this theorem is a formalized adaptation of the central argument in [4], and will not be given here.

4. Homma's theorem. Homma [4] proved

Homma's THeOREM. Let $M^{n}, \tilde{M}^{n}$ and $\tilde{P}^{k}$ be two finite combinatorial n-manifolds (possibly with boundary) and a finite simplicial complex such that $\widetilde{M}^{n}$ is topologically embedded in $M^{n}, \widetilde{P}^{k}$ is piecerwise linearly embedded in $\operatorname{int}\left(\tilde{M}^{n}\right)$ and $2 k+2 \leqq n$. Then for any $\epsilon>0$ there is an $\epsilon-$ 
homeomorphism $h$ of $M^{n}$ onto itself such that

$$
h / M^{n}-U_{\bullet}\left(\tilde{P}^{k}\right)=1 \text {, }
$$

and

$$
h / \tilde{P}^{k}: \tilde{P}^{k} \rightarrow M^{n}
$$

is piecewise linear.

Note that the "niceness" condition on $\tilde{P}^{k} \subset M^{n}$ requires $\tilde{P}^{k}$ to appear piecewise linearly embedded in some triangulation of a neighborhood $\tilde{M}^{n}$ of itself. The condition is therefore "global" with respect to $\widetilde{P}^{k}$, and this, together with the fact that $h$ is not given isotopic to the identity, is the main difference between Homma's theorem and Theorem 1.1.

Homma's technique appears in the proof of Theorem 3.4, and this puts us in a position to prove

THEOREM 4.1. Let the following be given:

(1) $M^{n}$, a possibly noncompact combinatorial n-manifold,

(2) $\tilde{M}^{n}$, a possibly noncompact combinatorial n-manifold, topologically embedded in $M^{n}$,

(3) $\widetilde{P}^{k}, a$ possibly infinite simplicial complex, piecewise linearly embedded as a closed subset of $\tilde{M}^{n}$,

(4) $\tilde{L}$, a subcomplex of $\tilde{P}^{k}$ such that the closure of $\tilde{P}^{k}-\tilde{L}$ is a finite complex, and such that $\tilde{L}$ is piecerwise linearly embedded in $M^{n}$ as well as in $\tilde{M}^{n}$.

If $2 k+2 \leqq n$, then for any $\epsilon>0$ there is an $\epsilon$-push $h$ of $\left(M^{n}, \tilde{P}^{k}-\tilde{L}\right)$ such that

$$
h / \widetilde{P}^{k}: \widetilde{P}^{k} \rightarrow M^{n}
$$

is piecewise linear, and

$$
h / \tilde{L}=1 .
$$

Let $\operatorname{Hom}\left(\tilde{P}^{k}, \tilde{P}^{k}-\tilde{L} ; \tilde{M}^{n}\right)$ denote the set of all embeddings $f: \widetilde{P}^{k} \rightarrow \widetilde{M}^{n}$ which restrict to the inclusion on $\tilde{L}$.

Let $F$ denote the set of piecewise linear embeddings of $\tilde{P}^{k}$ into $\tilde{M}^{n}$ which restrict to the inclusion on $L$. Among these is the inclusion $i: \widetilde{P}^{k} \subset \widetilde{M}^{n}$. Because $2 k+2 \leqq n$, general position arguments $[1 ; 4]$ prove that $F$ is dense and solvable in $\operatorname{Hom}\left(\widetilde{P}^{k}, \widetilde{P}^{k}-\tilde{L} ; \tilde{M}^{n}\right)$.

Since $\widetilde{M}^{n}$ is an open subset of $M^{n}$, it has a triangulation $\hat{M}^{n}$ which makes the inclusion $\hat{M}^{n} \subset M^{n}$ piecewise linear. Let $F^{\prime}$ denote the set of piecewise linear embeddings of $\tilde{P}^{k}$ into $\hat{M}^{n}$ which restrict to the inclusion on $\tilde{L}$. Then $F^{\prime}$ is also dense and solvable in $\operatorname{Hom}\left(\widetilde{P}^{k}, \widetilde{P}^{k}-\tilde{L} ; \widetilde{M}^{n}\right)$. 
Now by Theorem 3.4, $F \cup F^{\prime}$ is dense and solvable.

Using the denseness of $F^{\prime}$, first find an embedding $f^{\prime} \in F^{\prime}$ within $\delta_{F \cup F^{\prime}}(\epsilon)$ of the embedding $i$. Then because $F \cup F^{\prime}$ is solvable, we get an $\epsilon$-push $h$ of $\left(\tilde{M}^{n}, \widetilde{P}^{k}-\tilde{L}\right)$ such that

$$
h i=f^{\prime} \text {, }
$$

which means that

$$
h / \widetilde{P}^{k}: \widetilde{P}^{k} \rightarrow \hat{M}^{n} \subset M^{n}
$$

is piecewise linear. For sufficiently small $\epsilon, h$ will extend via the identity to an $\epsilon$-push of $\left(M^{n}, \widetilde{P}^{k}-\tilde{L}\right)$. Since $h i=f^{\prime}$ implies $h / \tilde{L}=1$, this completes the proof of Theorem 4.1, and therefore also of Homma's theorem.

5. Taming locally tame embeddings. In this section we use Theorem 4.1 to prove Theorem 1.1 with the temporary restriction: the triangulation of $P^{k}$ must be one in terms of which $f$ is locally tame. This restriction will be removed in the next section.

Referring back to the definition of "locally tame," call a subset $A \subset P^{k}$ small if there is a neighborhood $U$ of $A$ in $P^{k}$ and a neighborhood $V$ of $f(A)$ in $M^{n}$ such that

(1) $f(U)=V \cap f\left(P^{k}\right)$,

(2) $V$ has a triangulation as a combinatorial manifold in terms of which $f / U: U \rightarrow V$ is piecewise linear with respect to an induced triangulation of $U$ as an open subset of $P^{k}$.

Observe that a finite disjoint union of compact small subsets of $P^{k}$ is itself small, the neighborhoods $U$ and $V$ for the union simply being disjoint unions of sufficiently small corresponding neighborhoods for the individual sets.

Taking a preliminary subdivision if necessary, assume $P^{k}$ to be triangulated so finely that the stars of closed simplexes are small. Let $N_{i}$ denote the closed regular neighborhood of $P^{i}$, the $i$-skeleton of $P^{k}$, in the second barycentric subdivision of $P^{k}$. Then

$$
N_{0} \subset N_{1} \subset \cdots \subset N_{k}=P^{k},
$$

and using the above observation it is easily verified that $N_{0}$ is small and that $\mathrm{Cl}\left(N_{i}-N_{i-1}\right)$, the closure of $N_{i}-N_{i-1}$, is small for $i=1, \cdots, k$.

The plan is to define, with the help of Theorem 4.1, $k+1$ homeomorphisms of $M^{n}$,

$$
h_{0}, h_{1}, \cdots, h_{k} \text {, }
$$

with the properties: 
(i) $h_{0}$ is an $(\epsilon / k+1)$-push of $\left(M^{n}, f\left(N_{0}\right)\right)$ such that $h_{0} f / N_{0}: N_{0} \rightarrow M^{n}$ is piecewise linear,

(ii) $h_{i}$ is an $(\epsilon / k+1)$-push of $\left(M^{n}, h_{i-1} h_{i-2} \cdots h_{0} f\left(\mathrm{Cl}\left(N_{i}-N_{i-1}\right)\right)\right)$ such that $h_{i} h_{i-1} \cdots h_{0} f / N_{i}: N_{i} \rightarrow M^{n}$ is piecewise linear, for $i=1, \cdots, k$.

Setting $h=h_{k} h_{k-1} \cdots h_{0}$ will then complete the proof.

STEP 0. Since $N_{0}$ is small, there is a neighborhood $U_{0}$ of $N_{0}$ in $P^{k}$ and a neighborhood $V_{0}$ of $f\left(N_{0}\right)$ in $M^{n}$ satisfying conditions (1) and (2) above. These neighborhoods are highly disconnected-they will generally have as many components as there are vertices in $P^{k}$-but this in no way affects the argument. To apply Theorem 4.1 , let

$M^{n}=M^{n}$ with its given combinatorial triangulation,

$\tilde{M}^{n}=V_{0}$, triangulated so that $f / U_{0}: U_{0} \rightarrow V_{0}$ is piecewise linear,

$\widetilde{P}^{k}=f\left(N_{0}\right)$, with the triangulation carried over from $N_{0}$ by $f$,

$\tilde{L}=\varnothing$, the empty set,

$\epsilon \leqq$ the present $\epsilon / k+1$.

Theorem 4.1 then asserts the existence of an $(\epsilon / k+1)$-push $h_{0}$ of $\left(M^{n}, f\left(N_{0}\right)\right)$ such that

$$
h_{0} f / N_{0}: N_{0} \rightarrow M^{n}
$$

is piecewise linear. This completes Step 0.

STEP 1. Since $\mathrm{Cl}\left(N_{1}-N_{0}\right)$ is small, there is a neighborhood $U_{1}$ of $\mathrm{Cl}\left(N_{1}-N_{0}\right)$ in $P^{k}$ and a neighborhood $V_{1}$ of $f\left(\mathrm{Cl}\left(N_{1}-N_{0}\right)\right)$ in $M^{n}$ satisfying conditions (1) and (2) above. To apply Theorem 4.1, let $M^{n}=M^{n}$ with its given combinatorial triangulation,

$\tilde{M}^{n}=h_{0}\left(V_{1}\right)$, triangulated so that $h_{0} f / U_{1}: U_{1} \rightarrow h_{0}\left(V_{1}\right)$ is piecewise linear,

$\tilde{P}^{k}=h_{0} f\left(N_{1} \cap U_{1}\right)$, with the triangulation carried over from $N_{1} \cap U_{1}$ by $h_{0} f$,

$\mathcal{L}=h_{0} f\left(N_{0} \cap U_{1}\right)$,

$\epsilon \leqq$ the present $\epsilon / k+1$.

Theorem 4.1 then asserts the existence of an $(\epsilon / k+1)$-push $h_{1}$ of $\left(M^{n}, h_{0} f\left(\mathrm{Cl}\left(N_{1}-N_{0}\right)\right)\right)$ such that

$$
h_{1} h_{0} f / N_{1}: N_{1} \rightarrow M^{n}
$$

is piecwise linear, provided $\epsilon$ is chosen so small that $h_{1}$ restricts to the identity outside $h_{0}\left(V_{1}\right)$. This completes Step 1.

Steps 2 through $\mathrm{k}$ are then performed in this manner, and the proof is completed by setting $h=h_{k} h_{k-1} \cdots h_{0}$.

6. Proof of Theorems 1.1 and 1.2. Let $\operatorname{Hom}\left(P^{k}, M^{n}\right)$ denote the set of all locally tame embeddings of $P^{k}$ into $M^{n}$, and let $2 k+2 \leqq n$. 
Let $\left(P^{k}\right)_{1}$ and $\left(P^{k}\right)_{2}$ denote $P^{k}$ with two different triangulations, and let $M^{n}$ have a fixed but arbitrary combinatorial triangulation. $F_{1}$ will denote the set of piecewise linear embeddings of $\left(P^{k}\right)_{1}$ into $M^{n}$, and $F_{1}^{\prime}$ the set of embeddings of $P^{k}$ into $M^{n}$ which are locally tame with respect to the triangulation $\left(P^{k}\right)_{1}$. Similarly for $F_{2}$ and $F_{2}^{\prime}$ with respect to $\left(P^{k}\right)_{2} . F_{1}$ and $F_{2}$ are each dense and solvable in $\operatorname{Hom}\left(P^{k}, M^{n}\right)$ by $[1 ; 4]$. Therefore by Theorem 3.3 and the modified version of Theorem 1.1 proved in the preceding section, $F_{1}^{\prime}$ and $F_{2}^{\prime}$ are also dense and solvable in $\operatorname{Hom}\left(P^{k}, M^{n}\right)$.

Now by Theorem 3.4, $F_{1}^{\prime} \cup F_{2}$ is dense and solvable. Let $f_{1}^{\prime} \in F_{1}^{\prime}$. Since $F_{2}$ is dense in $\operatorname{Hom}\left(P^{k}, M^{n}\right)$, choose $f_{2} \in F_{2}$ so that $d\left(f_{1}^{\prime}, f_{2}\right)$ $\left\langle\delta_{F^{\prime} \cup F^{\prime}}(\epsilon)\right.$. Then there is an $\epsilon$-push $h$ of $\left(M^{n}, f_{1}^{\prime}\left(P^{k}\right)\right)$ such that $h f_{1}^{\prime}=f_{2}$. Since $f_{1}^{\prime}$ was locally tame with respect to $\left(P^{k}\right)_{1}$ while $f_{2}$ is piecewise linear with respect to $\left(P^{k}\right)_{2}$, this completes the proof of Theorem 1.1.

As a corollary to Theorem 1.1, we get

THEOREM 6.1. Let $f$ be an embedding of the polyhedron $P^{k}$ into the combinatorial manifold $M^{n}$. If $2 k+2 \leqq n$ and $f$ is locally tame with respect to one triangulation of $P^{k}$, then $f$ is locally tame with respect to any triangulation of $P^{k}$.

But then $\operatorname{Hom}\left(P^{k}, M^{n}\right)=F_{1}^{\prime}$, which is solvable, and Theorem 1.2 follows.

7. Proof of Theorem 1.3. Now, since $\operatorname{Hom}\left(P^{k}, M^{n}\right)$ is solvable when $2 k+2 \leqq n$, let $\delta=\delta(1)$ be determined according to this solvability. Suppose that $f, f^{\prime} \in \operatorname{Hom}\left(P^{k}, M^{n}\right)$ are homotopic as maps of $P^{k}$ into $M^{n}$. Then there is a sequence of continuous maps

$$
f=g_{0}, g_{1}, \cdots, g_{r}=f^{\prime}
$$

of $P^{k}$ into $M^{n}$ such that $d\left(g_{i}, g_{i+1}\right)<\delta$. Hom $\left(P^{k}, M^{n}\right)$, since it includes the piecewise linear embeddings, is dense in the mapping space $\left(M^{n}\right)^{P^{k}}$, and hence there is also a sequence of elements of $\operatorname{Hom}\left(P^{k}, M^{n}\right)$,

$$
f=f_{0}, f_{1}, \cdots, f_{r}=f^{\prime},
$$

such that $d\left(f_{i}, f_{i+1}\right)<\delta$. According to the definition of $\delta$, there exist 1 -pushes $h_{i}$ such that $h_{i} f_{i-1}=f_{i}$, for $i=1, \cdots, r$. Then

$$
h=h_{r} h_{r-1} \cdots h_{1}
$$

is a homeomorphism of $M^{n}$ onto itself which is isotopic to the identity, such that

proving Theorem 1.3.

$$
h f=f^{\prime},
$$


8. Applications. An embedding $f$ of a topological manifold $M^{k}$ into a topological manifold $M^{n}$ is said to be locally flat if for each $x \in M^{k}$ there is a neighborhood $U$ of $f(x)$ in $M^{n}$ such that the pair $\left(U, U \cap f\left(M^{k}\right)\right)$ is homeomorphic to the pair $\left(R^{n}, R^{k}\right)$.

THEOREM 8.1. Let $f$ be a locally fat embedding of the closed combinatorial manifold $M^{k}$ into the combinatorial manifold $M^{n}$. If $2 k+2 \leqq n$, then for each $\epsilon>0$ there is an $\epsilon$-push $h$ of $\left(M^{n}, f\left(M^{k}\right)\right)$ such that

$$
h f: M^{k} \rightarrow M^{n}
$$

is piecewise linear.

For each $x \in M^{k}$, choose a neighborhood $U$ of $f(x)$ in $M^{n}$ as in the above definition. $U \cap f\left(M^{k}\right)$ inherits a triangulation as an open combinatorial manifold from $M^{k}$ via $f$. Since $\left(U, U \cap f\left(M^{k}\right)\right)$ is homeomorphic to $\left(R^{n}, R^{k}\right)$, this triangulation extends to a "product" triangulation of $U$ as a combinatorial manifold, in terms of which $f / f^{-1}\left(U \cap f\left(M^{k}\right)\right)$ is piecewise linear. But then $f$ is locally tame, and an application of Theorem 1.1 completes the proof.

THEOREM 8.2. Let $f$ and $f^{\prime}$ be locally tame embeddings of the polyhedron $P^{k}$ into the $n$-sphere $S^{n}$ (or Euclidean space $R^{n}$ ). If $2 k+2 \leqq n$, there is a homeomorphism $h$ of $S^{n}\left(\right.$ or $\left.R^{n}\right)$ onto itself which is isotopic to the identity, such that $h f=f^{\prime}$.

For $f$ must be homotopic to $f^{\prime}$, and then Theorem 1.3 applies.

A special case of this was given in [5].

\section{REFERENCES}

1. V. K. A. M. Gugenheim, Piecewise linear isotopy and embedding of elements and spheres. I, II, Proc. London Math. Soc. (3) 3 (1953), 29-53; 129-152.

2. H. Whitney, Differentiable manifolds, Ann. of Math. (2) 37 (1936), 645-680.

3. M. Brown, (to appear).

4. T. Homma, On the imbedding of polyhedra in manifolds, (to appear).

5. H. Gluck, Unknotting $S^{1}$ in $S^{4}$, Bull. Amer. Math. Soc. 69 (1963), 91-94.

HARVARD UNIVERSITY 